\title{
Interações medicamentosas relacionadas a associações de antimicrobianos em um hospital de média complexidade
}

\author{
Drug interactions related to antimicrobial associations in a medium complexity hospital
Interacciones farmacológicas relacionadas con asociaciones antimicrobianas en un hospital de complejidad media

Felipe Andrade Gaspar ${ }^{1}$, Daniel Tarciso Martins Pereira ${ }^{1 *}$, Betânia Braga da Silva², Hidelbrando Ferreira Rodrigues ${ }^{1}$.

\section{RESUMO}

Objetivo: Identificar potenciais interações medicamentosas, ocasionadas pelo uso concomitante de mais de um antimicrobiano, que possam comprometer a segurança de pacientes internados em um hospital de média complexidade. Métodos: Tratou-se de um estudo transversal, retrospectivo, realizado mediante análise de prescrições de antimicrobianos dispensados a pacientes internados em unidade hospitalar, cujos dados coletados foram analisados e interpretados por meio de estatística descritiva e teste qui-quadrado, após aprovação pelo CEP-UFAM (CAAE 01581318.2.0000.5020). Resultados: As variáveis demonstraram prevalência de internações na clínica médica, pacientes do gênero feminino, prescrições de beta-lactâmicos e interações medicamentosas associadas a prescrição combinada de antimicrobianos. Identificou-se, ainda que as interações medicamentosas ocorreram principalmente em razão de erros de prescrição e pela não adesão aos protocolos clínicos institucionais. Conclusão: Portanto, faz-se necessário consolidar as ações da Comissão de Controle de Infecção Hospitalar $(\mathrm{CClH})$, buscando-se reduzir o uso irracional de antimicrobianos, e consequentemente, ampliar os padrões de segurança para aos pacientes.

Palavras-chave: Antimicrobianos, Interações medicamentosas, Hospital geral.

\begin{abstract}
Objective: This study sought to identify potential drug interactions caused by the concomitant use of more than one antimicrobial that could compromise the safety of patients admitted to a medium-complexity hospital. Methods: This was a cross-sectional, retrospective study, carried out by analyzing the prescriptions of antimicrobials dispensed to patients hospitalized in a hospital, whose collected data were analyzed and interpreted using descriptive statistics and chi-square test, after approval by the CEP-UFAM (CAAE 01581318.2.0000.5020). Results: The variables showed a prevalence of hospitalizations in the medical clinic, female patients, beta-lactam prescriptions and drug interactions associated with the combined prescription of antimicrobials. It was also identified that drug interactions occurred mainly due to prescription errors and nonadherence to institutional clinical protocols. Conclusion: Therefore, it is necessary to consolidate the actions of the Hospital Infection Control Commission $(\mathrm{CClH})$, seeking to reduce the irrational use of antimicrobials, and consequently, to expand the safety standards for patients.
\end{abstract}

Key words: Antimicrobials, Drug interactions, General hospital.

\section{RESUMEN}

Objetivo: Este estudio buscó identificar posibles interacciones medicamentosas provocadas por el uso concomitante de más de un antimicrobiano que pudieran comprometer la seguridad de los pacientes ingresados en un hospital de complejidad media. Métodos: Estudio transversal, retrospectivo, realizado mediante el análisis de las prescripciones de antimicrobianos dispensadas a pacientes hospitalizados en un

\footnotetext{
1 Universidade Federal do Amazonas (UFAM), Itacoatiara - AM. *E-mail: dtarciso@ufam.edu.br

2 Hospital Regional José Mendes, Itacoatiara - AM.
} 
hospital, cuyos datos recolectados fueron analizados e interpretados mediante estadística descriptiva y prueba de chi-cuadrado, previa aprobación del CEP-UFAM (CAAE 01581318.2.0000.5020). Resultados: Las variables mostraron una prevalencia de hospitalizaciones en la clínica médica, pacientes del sexo femenino, prescripción de betalactámicos e interacciones medicamentosas asociadas a la prescripción combinada de antimicrobianos. También se identificó que las interacciones farmacológicas se produjeron principalmente debido a errores de prescripción y al incumplimiento de los protocolos clínicos institucionales. Conclusión: Por lo tanto, es necesario consolidar las acciones de la Comisión de Control de Infecciones Hospitalarias $(\mathrm{CCIH})$, buscando reducir el uso irracional de antimicrobianos y, en consecuencia, ampliar los estándares de seguridad para los pacientes.

Palabras clave: Antimicrobianos, Interacciones farmacológicas, Hospital general.

\section{INTRODUÇÃO}

As Interações Medicamentosas (IMs) são respostas farmacológicas ocasionadas pelo uso concomitante de mais de um medicamento, causando mudança quantitativa ou qualitativa em seus efeitos (ZIEHL EA, et al., 2016). Trata-se de um problema de saúde pública que eleva o ônus econômico associado a assistência médica em pacientes internados, cujo desfecho pode conduzir a eventos adversos graves ou falhas terapêuticas (BARBOSA KL e MEDEIROS KCS, 2018; ISMAIL M, et al., 2018).

Nas últimas décadas a terapêutica antimicrobiana tem despertado especial interesse no tocante ao tratamento e erradicação das doenças infecciosas e consequente aumento da expectativa de vida da população mundial. Em oposição, o uso abusivo e desnecessário desses agentes não somente afeta os pacientes que os utilizam, mas também o ambiente hospitalar ao promover o surgimento de resistência microbiana que resulta da pressão seletiva exercida sobre a microbiota (SILVA JO e PAIXÃO JA, 2021; DE LARA VIEIRA PJ e DE FREITAS LT, 2021).

$\mathrm{Na}$ atualidade, a resistência microbiana constitui-se um grave problema de saúde mundial, que aumenta a morbimortalidade nos serviços de saúde em geral (REIS HPLC, et al., 2013). Várias evidências têm demonstrado o papel das mutações cromossômicas e plasmídeos como mecanismos naturais que conduzem ao aumento progressivo da resistência microbiana, acelerada em função do uso indiscriminado de antimicrobianos (DURÁN L, 2018).

No Brasil, $14 \%$ de todas as internações estão relacionadas as infecções hospitalares, classificadas como problemas relacionados à assistência de saúde. Dito isto, o ambiente hospitalar, com sua diversidade de pacientes com patologias distintas, elevado tempo de internação, procedimentos invasivos, favorece 0 surgimento da infecção hospitalar e de microrganismos multirresistentes (SILVA JO e PAIXÃO JA, 2021).

Meio milhão de pessoas morrem anualmente por infecções ocasionadas por microrganismos multirresistentes, segundo projeções do governo britânico. Por conseguinte, observa-se forte impacto nas economias e sistemas de saúde de diferentes países, a exemplo dos EUA que anualmente gastam cerca de 35 bilhões de dólares no tratamento destas infecções. Ademais, é fato comum, que pacientes internados em serviços de saúde, possuem maior tendência a desenvolver interações medicamentosas em função dos fatores idade, número de medicamentos prescritos, gravidade do quadro clínico, bem como comorbidades associadas (KRAEMER SA, et al., 2019; HAN X, et al., 2018).

Em 2015, a Organização Mundial de Saúde (OMS) aprovou um plano de ação global com o objetivo de compreender os padrões locais de resistência microbiana a medicamentos (WORLD HEALTH ORGANIZATION (WHO), 2020). Por conseguinte, melhorar a racionalização no uso e na redução do risco potencial de IMs, representa um grande desafio da terapia antimicrobiana na atualidade. Neste contexto, o uso da tecnologia da informação em medicamentos e a educação continuada para equipes multiprofissionais, são ferramentas importantes para o monitoramento de processos e ajudam a evitar erros (TRENTIN KM e RENNER JPD, 2019).

O controle da resistência microbiana depende da constatação da efetivação de medidas de controle de infecção hospitalar; da atualização dos currículos dos cursos de graduação em saúde em relação ao 
diagnóstico, manejo e terapêutica de infecções prevalentes; da promoção do uso racional de antimicrobianos em conformidade com as ações da Comissão de Controle de Infecção Hospitalar (CCIH) quanto a garantia dos padrões de eficácia, segurança e qualidade para o paciente (HOLLOWAY KA, et al., 2016).

É importante destacar que os estudos epidemiológicos de utilização de medicamentos permitem conhecer quais são os fatores e/ou processos mais críticos que influenciam o surgimento e a disseminação da resistência microbiana e, por conseguinte, propor ações necessárias para detecção, prevenção e gestão do uso irracional de antimicrobianos em serviços de saúde (ALLCOCK S, et al., 2017).

Assim, este estudo buscou identificar a frequência de IMs que pode comprometer a segurança de pacientes internados em uso associado de antimicrobianos em uma unidade hospitalar de média complexidade. Adicionalmente, sugeriu quais ações são necessárias para o enfrentamento do uso irracional e prevenção do risco potencial de IMs ocasionadas por antimicrobianos em serviços de saúde.

\section{MÉTODOS}

Trata-se de um estudo quantitativo, retrospectivo, do tipo transversal, baseado na análise de prescrições de antimicrobianos dispensados a pacientes internados em um hospital geral de média complexidade, situado no interior do Amazonas.

Para tanto, escolheu-se como fonte de dados 329 prescrições de antimicrobianos, datadas entre março e maio de 2018, e dispensadas pelo Serviço de Farmácia Hospitalar a pacientes internados nas clínicas médica, cirúrgica, pediátrica e obstétrica. As informações constituíram a base de dados (idade, gênero, unidade de internação hospitalar, farmacoterapia antimicrobiana, etc.), cuja análise estatística das variáveis foi realizada no pacote estatístico Action Stat Versão Acadêmica, considerando o nível de significância de $5 \%$ e poder de $95 \%$. A análise das interações medicamentosas foi realizada a partir da plataforma Micromedex®. Os aspectos éticos deste estudo foram apreciados e aprovados pelo Comitê de Ética da Universidade Federal do Amazonas, após submissão em 28/09/2018 via Plataforma Brasil CAAE (01581318.2.0000.5020).

\section{RESULTADOS}

Neste estudo, foram apresentados os dados referentes a análise de 329 prescrições de antimicrobianos coletadas no período de março a maio de 2018, cuja média de medicamentos totais por número de prescrições foi de 1,19 ( $D P \pm 0,5)$. Por sua vez, a maioria dos pacientes internados possui gênero feminino $(60,2 \% ; n=198)$, com idade média de 25 anos.

A análise das frequências relacionadas ao número de pacientes atendidos $x$ Unidade de Internação Hospitalar $(\mathrm{UIH})$ demostrou que a clínica médica responde por $34,6 \%$ das internações trimestrais, seguido pela clínica obstétrica, pediátrica e cirúrgica (Tabela 1). Estabelecido o teste $\mathrm{X} 2=65,45$ ( $p$-valor = 4,01x1014), rejeitamos a hipótese nula, e comprovamos que existe dependência entre o gênero do paciente e a clínica onde foi prescrito o tipo de antimicrobiano 1, ao nível de significância de 5\%.

Tabela 1 - Frequência de pacientes atendidos por UIH.

\begin{tabular}{|c|c|c|c|c|}
\hline \multirow{2}{*}{ UIH } & \multicolumn{2}{|c|}{$\mathbf{n}$} & \multicolumn{2}{|c|}{$\%$} \\
\hline & $\hat{0}$ & 9 & $\hat{0}$ & 9 \\
\hline Clínica Obstétrica & 0 & 73 & 0,00 & 22,1 \\
\hline Clínica Médica & 52 & 62 & 15,8 & 18,8 \\
\hline Clínica Pediátrica & 37 & 34 & 11,2 & 10,3 \\
\hline Clínica Cirúrgica & 42 & 29 & 12,7 & 8,8 \\
\hline
\end{tabular}

Fonte: Gaspar FA, et al., 2021.

No total, foram prescritos 393 antimicrobianos, cujas classes farmacológicas de maior frequência foram beta-lactâmicos $(79,1 \% ; n=311)$, nitroimidazólicos $(8,1 \% ; n=32)$ e aminoglicosídeos $(5,1 \% ; n=20)$. Em comum, observou-se discrepância quanto a variação e frequência de antimicrobianos prescritos por UIH, exceto pela analogia da prescrição de agentes beta beta-lactâmicos, com frequência superior a 67\% (Tabela 2). 
Tabela 2 - Frequência dos principais antimicrobianos prescritos por UIH.

\begin{tabular}{lcc}
\hline UlH x antimicrobiano & $\mathbf{n}$ & $\%$ \\
\hline Clínica Obstétrica & 2 & 2,6 \\
\hline Aminoglicosídeos & 73 & 94,8 \\
B-Lactâmicos & 1 & 1,3 \\
Macrolídeos & 1 & 1,3 \\
Nitroimidazólicos & 77 & 100 \\
\hline Total & & \\
\hline Clínica Médica & 1 & 0,7 \\
\hline Aminoglicosídeos & 101 & 75,9 \\
B-Lactâmicos & 1 & 0,7 \\
Glicopeptídeos & 1 & 0,7 \\
Licosamidas & 6 & 4,5 \\
Macrolídeos & 10 & 7,5 \\
Nitroimidazólicos & 7 & 5,2 \\
Quinolonas & 6 & 4,5 \\
Sulfonamidas & 133 & 100 \\
\hline Total & & \\
\hline Clínica Pediátrica & 9 & 10,5 \\
\hline Aminoglicosídeos & 70 & 82,3 \\
B-Lactâmicos & 2 & 2,3 \\
Macrolídeos & 4 & 4,7 \\
Nitroimidazólicos & 85 & 100 \\
\hline Total & & \\
\hline Clínica Cirúrgica & 8 & 8,1 \\
\hline Aminoglicosídeos & 66 & 67,3 \\
B-Lactâmicos & 6 & 6,1 \\
Licosamidas & 1 & 1,0 \\
Macrolídeos & 17 & 17,3 \\
Nitroimidazólicos & 98 & 100 \\
\hline Total & &
\end{tabular}

Fonte: Gaspar FA, et al., 2021.

A análise, tendo por parâmetro o total de prescrições, demonstrou que 5,9\% ( $n=19)$ são combinações de 8 diferentes classes de antimicrobianos, com incidência de $63,1 \%(n=12)$ de interações medicamentosas com associações de antimicrobianos classificadas quanto à gravidade (Tabela 3 ). Considerando o total de prescrições, nota-se que a IM entre beta-lactâmicos e aminoglicosídeos $(1,8 \%, n=6)$ foi a mais frequente e de menor gravidade. A análise da frequência de $\mathrm{IM}$ versus $\mathrm{UIH}$, demonstrou que a clínica pediátrica responde pelo maior índice de prescrições com IMs $(58,3 \%, n=7)$.

Tabela 3 - Frequência de IMs associadas a antimicrobianos por UIH - 2018.

\begin{tabular}{|c|c|c|c|c|c|}
\hline IM & Tipo/Mecanismo & $\begin{array}{l}\text { Sexo } \\
i / q\end{array}$ & Clínica & $\begin{array}{l}\text { Total } \\
\mathbf{N}\end{array}$ & $\begin{array}{c}\text { Frequência } \\
\text { Relativa } \\
\%\end{array}$ \\
\hline $\begin{array}{l}\text { Ciprofloxacino + } \\
\text { Metronidazol } \\
\end{array}$ & $\begin{array}{l}\text { Maior: Aumento do intervalo QT } \\
\text { em uso prolongado }\end{array}$ & q (2) & Médica (2) & 2 & 16,6 \\
\hline $\begin{array}{l}\text { Ampicilina + } \\
\text { Gentamicina }\end{array}$ & $\begin{array}{l}\text { Menor: Perda da eficácia dos } \\
\text { aminoglicosídeos em uso } \\
\text { concomitante }\end{array}$ & $\begin{array}{l}P(4) \\
\text { P }(4) \\
\mathcal{O}(1)\end{array}$ & Pediátrica (5) & 5 & 41,6 \\
\hline $\begin{array}{l}\text { Oxacilina }+ \\
\text { Gentamicina }\end{array}$ & $\begin{array}{l}\text { Menor: Perda da eficácia dos } \\
\text { aminoglicosídeos em uso } \\
\text { concomitante }\end{array}$ & $\widehat{\partial}(2)$ & Pediátrica (1) & 1 & 8,3 \\
\hline $\begin{array}{l}\text { Cefalotina }+ \\
\text { Gentamicina }\end{array}$ & Médio: Causam nefrotoxicidade & $\begin{array}{l}+(2) \\
\text { P (2) } \\
\text { O }(2) \\
\end{array}$ & $\begin{array}{r}\text { Pediátrica (1) } \\
\text { Cirúrgica (3) }\end{array}$ & 4 & 33,3 \\
\hline
\end{tabular}

Fonte: Gaspar FA, et al., 2021. 


\section{DISCUSSÃO}

O perfil assistencial na unidade locus da pesquisa demonstra uma população predominantemente feminina (60,2\%; n=198), com idade média de 25 anos, semelhante à observada em um Hospital Geral do Sul do Brasil (BORGES LS, et al., 2018). Em contrapartida, IMs em hospitais universitários possui prevalência de população masculina (51,2\% a 65\%), com idade média entre 52 - 63,6 anos (SILVA ASP e SILVA STF, 2020). No Brasil, culturalmente, as mulheres são mais preocupadas com o processo de saúde-doença e utilizam com mais frequência os serviços de saúde (COSTA CSC, et al., 2021). Por sua vez, os homens só recorrem aos serviços de saúde quando por doença em fase avançada ou associada a situações de emergência/urgência (BORGES LS, et al., 2018).

Estudo multicêntrico retrospectivo realizado na China demonstrou que a gravidade da doença na admissão, a idade e o subtratamento com antimicrobianos são fatores importantes para o óbito entre pacientes idosos hospitalizados com pneumonia adquirida na comunidade (PAC) (HAN X, et al., 2018). De modo complementar outros estudos têm demonstrado que pacientes idosos e com comorbidades crônicas consomem três vezes mais medicação que os jovens, e, por conseguinte, são os mais suscetíveis a desenvolver interações medicamentosas potenciais (BARBOSA KL e MEDEIROS KCS, 2018; SILVA ASP e SILVA STF, 2020). Consequentemente, o perfil de internações hospitalares trata-se de uma representação das necessidades e características socioeconômicas, culturais e fenotípicas da população deste estudo.

A elevada diversidade de casos da clínica médica remeteu a maior variabilidade de antimicrobianos prescritos, e, esteve em consonância com outros estudos que apontaram os antimicrobianos beta-lactâmicos os fármacos de escolha no tratamento profilático da maioria das infecções (HAN X, et al., 2018). Estudo realizado no Hospital Universitário (HU) da Universidade Federal de Sergipe indicou que existe correlação entre as classes de antimicrobianos e as especialidades clínicas, o tipo de infecção e a política de uso de antimicrobianos da instituição (DANTAS JO, et al., 2015).

Diversos estudos têm correlacionado a ampla prescrição de agentes antimicrobianos com os elevados gastos com medicamentos hospitalares. Estima-se que, em $50 \%$ dos casos, haja uso inapropriado de antimicrobianos em hospitais, cujo desfecho conduz ao desenvolvimento da resistência microbiana e aumento dos riscos de reações adversas a medicamentos (SILVA ERM, 2019).

Assim, o diagnóstico adequado; a escolha do fármaco, esquema posológico; e o tempo de terapêutica, são elementos importantes para o sucesso da terapêutica em infecções bacteriana (DE LARA VIEIRA PJ e DE FREITAS LT, 2021). Do mesmo modo, a terapia empírica deve se apoiar no diagnóstico laboratorial realizado a partir de amostras de microrganismos isolados do paciente (SILVA ERM, 2019). Portanto, a consequente falta da estruturação de Laboratório de Microbiologia na unidade hospitalar em estudo, contribui para o emprego de antimicrobianos em infecções não-sensíveis e doenças não-infecciosas, sob esquemas inadequados, favorecendo a resistência microbiana.

No contexto dos Hospitais Universitários brasileiros, revisão sistemática investigou o fenômeno das interações medicamentosas e descreveu médias de interações medicamentosas por prontuário de 0,70 no setor de Emergências Clínicas do Hospital São Paulo; 1,90 na Clínica Médica do Hospital Universitário da Universidade de São Paul e 3,04 na Unidade de Terapia Intensiva do Hospital Universitário do Ceará. Tal estudo apontou como possíveis fatores preditores associados à ocorrência de interações medicamentosa a falta de conhecimento da equipe multidisciplinar de saúde; a ausência ou dificuldades de acesso aos sistemas de informação, do tipo Micromedex ${ }^{\circledR}$ e o uso de medicamentos isentos de prescrição (MIP) por parte dos pacientes (SILVA ASP e SILVA STF, 2020).

Embora haja poucos estudos no Brasil, neste observou-se $3,6 \%(n=12)$ de prevalência de IMs em prescrições hospitalares com antimicrobianos. Estudo transversal, retrospectivo, realizado com pacientes internados na UTI de um hospital do Rio Grande do Sul, demonstra que a prevalência de IM em prescrições hospitalares com antimicrobianos foi da ordem de $25,8 \%(n=8)$, considerando 31 possíveis casos de IMs (NEVES C e COLET C, 2015). Na prática médica a combinação de antimicrobianos resulta em IMs benéficas ou desejáveis, com sinergia de efeito em infecções mono e polimicrobianas; aumento da adesão ao tratamento; prevenção da emergência de resistência bacteriana; e, redução de dosagens com menos efeitos tóxicos (SEHN R, et al., 2013). 
Contudo, a possibilidade de ocorrência de antagonismo químico soma-se as evidências de alto risco de efeitos secundários sem maior efetividade clínica, para o uso concomitante de beta-lactâmicos e aminoglicosídeos (SILVA ERM, 2019). Nosso estudo descreveu que 41,6\% ( $n=5)$ das IMs estão relacionadas ao uso associado de ampicilina e gentamicina na clínica pediátrica, amplamente recomendada para 0 tratamento empírico de sepse neonatal precoce (AAMIR M, et al., 2018). Estudos in vitro indicam que tal associação levam a inativação do aminoglicosídeo pelo beta-lactâmico (MOURAD AM, et al., 2017).

Ressalta-se ainda, neste estudo, a interação medicamentosa relacionada a terapia combinada de cefalotina e gentamicina ( $33,3 \% ; n=4)$ que conduz a uma ação nefrotóxica sinérgica, de gravidade moderada. Diversos estudos clínicos demonstraram dano renal atribuído possivelmente ao acúmulo de aminoglicosídeos nos lisossomas renais interferindo na atividade da adenosina trifosfatase dependente $\mathrm{Na}^{+} \mathrm{e} \mathrm{K}^{+}$, ao mesmo tempo que, as cefalosporinas ocasionam lesão mitocondrial com comprometimento da respiração celular (AFIUNE LAF, et al., 2016; SILVERBLATT F, 1982).

A combinação de quinolonas e nitroimidazólicos é amplamente utilizada no tratamento de infecções mistas aeróbias / anaeróbias ocasionadas por Escherichia coli, Staphylococcus aureus, Pseudomonas aeruginosa, Enterococcus faecalis, Clostridium perfringens e Clostridium difficile (OLUTUMBI AIM, et al., 2019). Entretanto, há IM na terapia combinada entre ciprofloxacino e metronidazol, cujo desfecho conduz ao prolongamento do intervalo QT, associado a arritmias fatais e parada cardíaca. Em nosso estudo, 16,6\% (n = 2) das IMs foram atribuídas a esta associação de antimicrobianos em pacientes da clínica médica, com risco superior ao observado entre pacientes de hospitais do Paquistão (KHAN Q, et al., 2019).

Pode-se notar a importância da polifarmácia e dos erros de prescrição como fatores de riscos associados à prevalência de IMs que se contrapõe a padronização da terapêutica, da adoção e do seguimento de protocolos clínicos a fim de se evitar as infecções bacterianas resistentes (DANTAS JO, et al., 2015; ZIMERMAN R, 2010). Diversos estudos têm comprovado que o advento de Programas de Otimização do Uso de Antimicrobianos (PROA) no âmbito hospitalar se traduz em uma série de benefícios, tais como, redução: de efeitos adversos e interações medicamentosas; da resistência microbiana; de infecções associadas aos cuidados de saúde em função do menor tempo de hospitalização; dentre outros (ROJAS-BONILLA M, et al., 2020).

Ressalta-se que, o sucesso inerente aos objetivos de um PROA está diretamente relacionado a adoção de esquemas curtos de tratamento fundamentados na eficácia, segurança e comodidade para o paciente; a otimização dos mecanismos de ação a partir de posologias modificadas de antimicrobianos, impedindo a resistência mutacional $\mathrm{e}$, o reversamente ou o uso combinado de antimicrobianos com respostas farmacológicas semelhantes e que atendam as necessidades clinicas do paciente (CARVALHO SL e DE OLIVEIRA AM, 2017).

Neste cenário, o advento das Comissões de Controle de Infecção Hospitalar $(\mathrm{CClH})$, com a obrigatoriedade da implementação de um programa de uso racional de antimicrobianos, soma-se a participação efetiva de farmacêuticos, cujas práticas da Atenção Farmacêutica (AF) aplicadas ao acompanhamento farmacoterapêutico tem contribuído para a redução dos indicadores de infecção hospitalar, interações medicamentosas e disseminação da resistência bacteriana nos serviços de saúde (SILVA JO e PAIXÃO JÁ, 2021; DE LARA VIEIRA PJ e DE FREITAS LT, 2021).

Como limitações deste estudo, não foi possível fomentar as relações de causa-efeito a partir da correlação medicamento prescrito versus patologia base, uma vez que não foi proposto a revisão do prontuário do paciente ou entrevista ao médico assistente, a fim de analisar outras variáveis que predispõem ao risco de ocorrência de IMs.

\section{CONCLUSÃO}

Neste estudo, foi possível identificar e analisar as IMs relacionadas as prescrições de associações de antimicrobianos dispensados a pacientes internados em uma unidade hospitalar de média complexidade. Pôde-se concluir que houve predomínio da terapia empírica e reduzida prevalência de IMs com esses 
agentes. Todavia, dado a importância da terapia antimicrobiana, torna-se indispensável a estruturação do laboratório de microbiologia para apoiar o diagnóstico clínico e reduzir a possibilidade de erros de prescrição. Por conseguinte, a promoção do uso racional de antimicrobianos deve estar amparada nas ações da Comissão de Controle de Infecção Hospitalar e na adoção de um Programa de Otimização do Uso de Antimicrobianos para minimizar a resistência microbiana e ampliar os padrões de segurança aos pacientes.

\section{AGRADECIMENTO}

Agradecemos a Secretaria Municipal de Saúde de Itacoatiara-AM pela anuência concedida, aos integrantes do Grupo de Pesquisa em Ciências Farmacêuticas e Saúde Pública (GPCFSP) da Universidade Federal do Amazonas que contribuíram de forma direta ou indireta para a realização deste estudo e a Fundação de Amparo à Pesquisa do Estado do Amazonas (FAPEAM) pela concessão da bolsa de no âmbito do Programa de Apoio à Iniciação Científica do Amazonas (PAIC-UFAM).

\section{REFERÊNCIAS}

1. AFIUNE LAF, et al. Potenciais interações medicamentosas em prescrições oriundas do hospital municipal e pronto socorro de Barra do Garças/MT. Revista Contexto \& Saúde, 2016; 16 (31): 128-138.

2. ALLCOCK S, et al. Antimicrobial resistance in human populations: challenges and opportunities. Global health, epidemiology and genomics, 2017; 2: 1-7.

3. AAMIR M, et al. Drug utilization in neonatal setting of Pakistan: focus on unlicensed and off label drug prescribing. BMC Pediatr, 2018; 18 (242): 1-8.

4. BARBOSA KL, MEDEIROS KCS. Interação medicamentosa: um agravo à saúde fragilizada. Revista de Atenção à Saúde, 2018; 16 (58): 84-92.

5. BORGES LS, et al. Estudo dos critérios determinantes de procura pelo serviço de emergência, por pacientes classificados como pouco urgentes e não urgentes, em um Hospital Geral do Sul do Brasil. Arquivos Catarinenses de Medicina, 2018; 47 (3): 60-73.

6. CARVALHO SF, DE OLIVEIRA AM. Impactos da legislação e a atuação do farmacêutico no uso racional de medicamentos sobre o combate a resistência bacteriana. Revista Acadêmica Oswaldo Cruz, 2017; 16: 1-12.

7. COSTA CSC, et al. Caracterização dos pacientes com diabetes mellitus tipo 2 em uma área de abrangência do programa saúde da família. Revista Eletrônica Acervo Enfermagem, 2021; 9: 1-7

8. DANTAS JO, et al. Avaliação da prescrição de antimicrobianos de uso restrito em um hospital universitário. Journal of Infection Control, 2015; 4 (2): 39-48.

9. DURÁN L. Resistencia antimicrobiana e implicancias para el manejo de infecciones del tracto urinario, Revista Médica Clínica Las Condes, 2018; 29 (2): 213-221.

10. HAN, X, et al. Effects of age, comorbidity and adherence to current antimicrobial guidelines on mortality in hospitalized elderly patients with community-acquired pneumonia. BMC infectious diseases, 2018; 18 (1): 1-11.

11. HOLLOWAY KA, et al. The impact of WHO essential medicines policies on inappropriate use of antibiotics. PLoS One, 2016: 11(3): 1-12.

12. ISMAIL M, et al. Potential drug-drug interactions in outpatient department of a tertiary care hospital in Pakistan: a cross-sectional study. BMC Health Services Research, 2018; 18: 762.

13. KHAN Q, et al. Prevalence of the risk factors for QT prolongation and associated drug-drug interactions in a cohort of medical inpatients. Journal of the Formosan Medical Association, 2019; 118: 109-115.

14. KRAEMER SA, et al. Antibiotic Pollution in the Environment: From Microbial Ecology to Public Policy. Microorganisms. 2019;7(6):180.

15. MOURAD AM, et al. Pharmaceutical care supported by the evidencing of "in vitro" interactions with the use of aminoglycosides and penicillins in patients affected by Enterococcus sp. African Journal of Pharmacy and Pharmacology, 2017; 11(22): 271-278.

16. NEVES C, COLET C. Perfil de uso de antimicrobianos e suas interações medicamentosas em uma UTI adulto do Rio Grande do Sul. Revista de Epidemiologia e Controle de Infecção, 2015; 5 (2): 65-71.

17. OLUTUMBI AIM, et al. In vitro influence of metronidazole on the activities of ciprofloxacin against clinically important bacterial isolates. GSC Biological and Pharmaceutical Sciences, 2019; 6 (3): 31-39

18. REIS HPLC, et al. Avaliação da resistência microbiana em hospitais privados de Fortaleza - Ceará. Revista Brasileira de Farmácia, 2013; 94 (1): 83-87.

19. ROJAS-BONILLA M, et al. Impact of an antimicrobial stewardship program in a pediatric third level hospital in Panama. Revista Chilena de Infectologia: Organo Oficial de la Sociedad Chilena de Infectologia, 2020; 37 (1): 9-18.

20. SEHN R, et al. Interações medicamentosas potenciais em prescrições de pacientes hospitalizados. Infarma - Ciências Farmacêuticas, 2013; 15 (9/10): 77-81.

21. SILVA ASP, SILVA STF. Aspectos qualitativos do estudo das interações medicamentosas em Hospitais Universitários brasileiros: revisão sistemática. VITTALLE - Revista de Ciências da Saúde, 2020; 32 (2); 109-120. 
22. SILVA ERM. Análise do perfil das prescrições de antimicrobianos na clínica médica de um hospital público do Pará. Revista Brasileira de Farmácia Hospitalar e Serviços de Saúde, 2019; 3 (2): 15-19.

23. SILVA JO, PAIXÃO JA Resistência bacteriana e a atuação do farmacêutico na promoção do uso racional de antibacterianos em âmbito hospitalar. Revista Artigos. Com. 2021; 29: 1-7.

24. SILVERBLATT F. Pathogenesis of nephrotoxicity of cephalosporins and aminoglycosides: a review of current concepts. Reviews of infectious diseases, 1982; 4 (2): S360-S365.

25. TRENTIN KM, RENNER JPD. Segurança do paciente e erros de medicação: uma revisão da literatura com ênfase no uso de antibióticos hospitalar. Anais do Seminário Científico do Programa de Pós-Graduação em Promoção da Saúde da UNISC, 2019.

26. ZIEHL EA, et al. Drug-drug interactions in an intensive care unit of a tertiary hospital in southern Chile: Evaluating databases agreement. Journal of Pharmacy \& Pharmacognosy Research, 2019; 7 (3): 184-192.

27. ZIMERMAN R. Uso indiscriminado de antimicrobianos e resistência microbiana. Brasília: Editora MS, $2010 ; 3: 12$.

28. WORLD HEALTH ORGANIZATION (WHO). Guidance for the surveillance of drug resistance in tuberculosis. 2020. 